\title{
Cardiopulmonary interactions during positive pressure ventilation
}

\author{
JOHN GRANTON MD \\ The Toronto Hospital, University of Toronto, Toronto, Ontario
}

J Granton. Cardiopulmonary interactions during positive pressure ventilation. Can Respir J 1996; 3(6):380-385.

Positive pressure ventilation (PPV) may lead to significant hemodynamic alterations. The cardiocirculatory effects of PPV occur through alterations in the loading conditions of the right and left ventricle and are mediated by changes in intrathoracic pressures and in lung volume. However, the net effect of PPV on cardiac output and hemodynamics is not always predictable. PPV may lead to either a decrease or an increase in cardiac performance. The cardiac consequences of PPV are also dependent on baseline loading conditions and contractile function of the heart.

Key Words: Positive end-expiratory pressure, Positive pressure ventilation
Interactions cardiopulmonaires pendant la ventilation par pression positive

RÉSUMÉ : La ventilation par pression positive (VPP) peut entraîner des modifications hémodynamiques importantes. Les effets cardiocirculatoires de la VPP se produisent par le biais de modifications des conditions de charge des ventricules gauche et droit et interviennent à la suite des changements dans les pressions intrathoraciques et dans le volume pulmonaire. Cependant, l'effet net de la VPP sur le débit cardiaque et sur l'hémodynamique n'est pas toujours prévisible. La VPP peut entraîner une diminution ou bien une augmentation de la fonction cardiaque. Les conséquences cardiaques de la VPP sont aussi dépendantes des conditions de charge de base et de la fonction contractile du coeur.
$\mathrm{P}$ ositive end-expiratory pressure (PEEP) is frequently used during positive pressure ventilation (PPV) to improve the oxygenation of patients with respiratory failure. However, PEEP often acts as a double-edged sword. While initially leading to an improvement in oxygenation, there comes a point when the adverse hemodynamic consequences of PEEP predominate and further increases are accompanied by a decrease in oxygen delivery. The effects of PEEP on cardiac performance occur largely through alterations in ventricular preload and afterload, and are mediated by changes in lung volume and pleural pressure (1). The mechanical loading conditions and contractile function of the heart set the stage on which PEEP's effects on preload and afterload will be played.

\section{OPTIMAL PEEP}

The net effect on PEEP may be determined by observing changes in systemic oxygen delivery $\left(\mathrm{DO}_{2}\right)$, the product of arterial oxygen content and cardiac output. $\left(\mathrm{DO}_{2}=\right.$ hemoglobin $\mathrm{X}$ arterial saturation $\mathrm{x}$ (stroke volume $\mathrm{x}$ heart rate).)

The concept of optimal PEEP stems from the observation that $\mathrm{DO}_{2}$ initially increases with an increase in PEEP due to an improvement in oxygenation (and in some patients an improvement in cardiac output). At some point, however, $\mathrm{DO}_{2}$ decreases due to an adverse effect of PEEP on stroke volume relative to an improvement in oxygenation (Figure 1). PEEP may affect the heart and great vessels at several anatomical levels and at different points of the cardiac cycle.

Corresondence: Dr John Granton, The Toronto Hospital, 11EN-213, 200 Elizabeth Street, Toronto, Ontario M5G 2C4. Telephone 416-340-4485, fax 416-340-3359, e-mail j.granton@utoronto.ca 


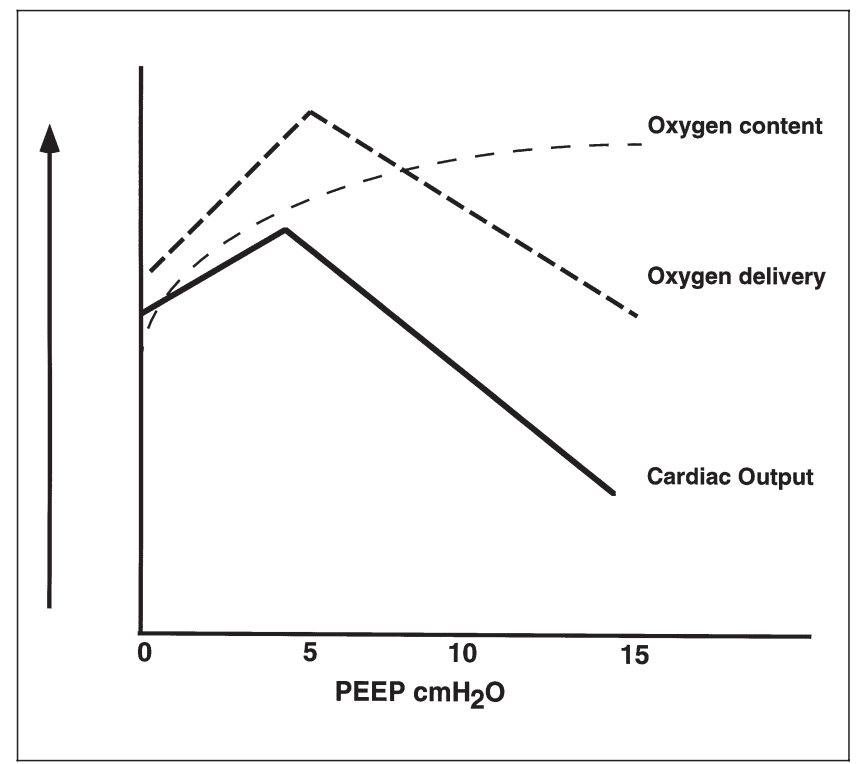

Figure 1) Relationship of oxygen delivery to positive end-expiratory pressure (PEEP). At low levels of PEEP, oxygen content is increased due to an increase in lung volume and improved ventilation-perfusion matching. Low levels of PEEP may also lead to an increase in cardiac output. The net effect is an increase in oxygen delivery. At higher levels of PEEP, cardiac output decreases and causes a reduction in oxygen delivery

The degree to which PEEP alters cardiac performance depends primarily on its ability to raise alveolar pressure and increase lung volume.

\section{PLEURAL AND ALVEOLAR PRESSURE}

At rest, pleural pressure is determined by the recoil pressure of the lung and outward movement of the chest wall and abdomen. During PEEP and PPV, alveolar pressure rises, the lung expands and pleural pressure increases. The degree of change in pleural pressure is dependent on the compliance of the lung and chest wall. A given level of alveolar pressure in a poorly compliant lung and/or a highly compliant thorax results in a small positive change in pleural pressure. Conversely, the same alveolar pressure in a compliant lung and/or a stiff thorax results in a larger positive increase in pleural pressure. To complicate matters, pleural pressure may not be equal in all regions of the chest. The pressure in dependent portions of the lung may be higher than in nondependent regions and juxtacardiac pressures may not be equal to those elsewhere in the thorax. Furthermore, in nonhomogeneous disease states (such as adult respiratory distress syndrome) where lung compliance is unevenly distributed, changes in lung volume (and hence pleural pressure) also are unevenly distributed for a given level of alveolar pressure.

\section{PERICARDIAL PRESSURE}

Changes in pleural pressure translate into a change in pericardial (juxtacardiac) pressure. However, pressure may not be transmitted equally to all regions surrounding the heart. With expansion of the lung some regions of the pericardium and heart may be directly compressed and distorted.

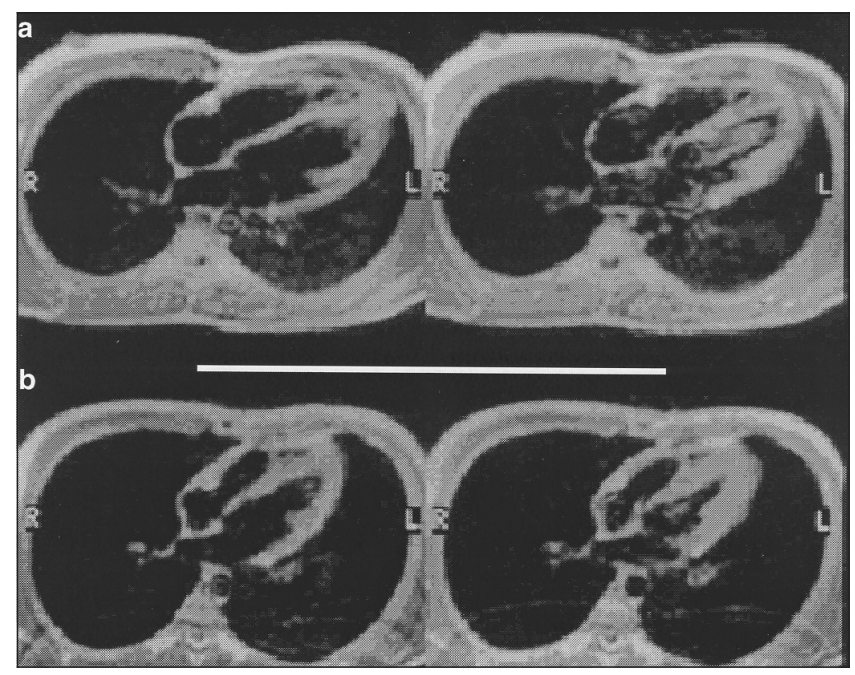

Figure 2) Magnetic resonance images of a coronal-saggital double-angulated projection illustrating the effects of continuous positive airway pressure (CPAP) on a normal volunteer's heart during end-diastole (left panels) and end-systole (right panels). a Images obtained during quiet breathing off CPAP. b Images obtained with the application of CPAP $15 \mathrm{~cm} \mathrm{H}_{2} \mathrm{O}$. There is obvious reduction in chamber size and loss of cardiac apical contact with the anterior chest wall. Reproduced with permission from reference 2

In addition, movement of the diaphragm may cause pericardial traction and chamber distortion. In a recent study (2) changes in ventricular dimensions during PEEP were evaluated using magnetic resonance imaging (Figure 2). During the application of $15 \mathrm{~cm} \mathrm{H}_{2} \mathrm{O}$ of PEEP, both left and right ventricular end-diastolic and end-systolic volumes decreased. The net effect was a decrease in right and left ventricular stroke volumes. This was associated with anterior rotation of the apex anteriorly and loss of contact with the anterior chest wall. The authors suggest that lung compression with PEEP resulted in the observed changes. However, this study did not evaluate the impact of a decrease in venous return, which may also have caused similar reductions in chamber sizes (see below). Thus, the relative importance of direct compressive effects by the lung remains to be clarified. Certainly in the setting of pericardial effusion or dilated cardiomyopathies direct compressive effects by the lung may become relevant to ventricular performance.

\section{STROKE VOLUME}

Changes in cardiac output caused by PPV and with the application of PEEP develop primarily though their relative effects on stroke volume and less frequently by reflex changes in heart rate. To examine the cardiac consequences of PPV and PEEP it is important to evaluate their influences on the determinants of stroke volume. Stroke volume is determined by three factors: preload, afterload and contractile function. The majority of investigations have focused on the effects of PPV on preload and afterload. For the sake of simplicity it is useful to consider the right and left ventricle separately. 


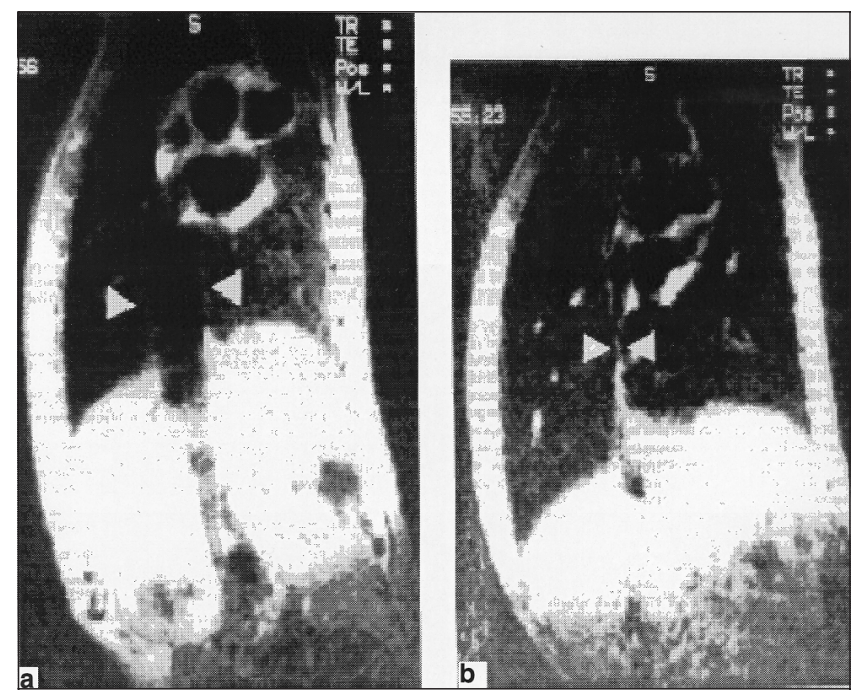

Figure 3) Magnetic resonance imaging of the thorax of a spontaneously breathing dog in the left lateral decubitus position. a Without positive end-expiratory pressure (PEEP), the inferior vena cava (arrows) has a uniform transverse diameter throughout its course. b The application of $\mathrm{PEEP}\left(10 \mathrm{~cm} \mathrm{H}_{2} \mathrm{O}\right)$ caused compression of the vena cava. Reproduced with permission from reference 4

\section{EFFECTS ON THE RIGHT VENTRICLE}

Right ventricular preload (venous return): The systemic reservoirs of the body consist of low resistance, high capacitance circuits that empty passively into the right atrium (3). The driving force for venous return is the difference between the systemic venous pressure and the right atrial pressure. With an increase in pleural pressure, the forces surrounding the great veins and right atrium increase. With a large increase in external pressure collapse of the great vessels may occur at some point along their course through the thorax. In fact, owing to an associated increase in abdominal pressure (descent of the diaphragm during PPV) the abdominal veins may also collapse. This has three practical implications. First, as downstream pressure increases, the gradient for venous return decreases and right ventricular preload falls. This in turn causes a decrease in stroke volume. Second, if the great veins collapse, the right atrial pressure may no longer provide the critical downstream pressure for venous return. The driving force for venous return therefore is no longer the pressure gradient between the systemic circulation and right atrium. Third, there is a need to reference intravascular pressure to the surrounding pressure. When measuring right atrial pressure (or any pressure that is determined using a pulmonary vascular catheter) a rise in pressure is often assumed to represent an increase in volume. However, with the addition of PEEP and PPV, this is clearly not the case.

In addition to its effects on pleural pressure, PPV may decrease venous return by direct compression of the great veins by the expanding lung. In a recent study in dogs, as PEEP was applied, a pressure drop was observed in a discrete portion of the inferior vena cava (4). This suggested localized compression of the inferior vena cava (Figure 3). The lung was observed via magnetic resonance imaging to expand and directly compress the thoracic vena cava. Others have also demonstrated that direct lung constraint of the ventricle may have significant effects during lung inflation by PEEP.

As mentioned earlier, PPV and PEEP may also lead to compression of intra-abdominal vascular structures. The importance of changes in intra-abdominal pressure on venous return is becoming increasingly appreciated. An early investigation in pigs demonstrated that PEEP could either increase venous return by increasing upstream driving pressure via direct compression of the splanchnic blood pool, or decrease venous return by increasing venous resistance by causing downstream vascular occlusion (5). Because the liver receives all of the splanchnic bloodflow (representing $40 \%$ of the total venous return), compression of the hepatic venous circulation or changes in sinusoidal pressure mediated by changes in intra-abdominal pressure with PEEP may also have important effects on venous return. Brienza et al (6) recently studied the effects of PEEP on liver arterial and venous bloodflows in an in vivo porcine mode. The application of PEEP resulted in a decrease in portal venous bloodflow due to an increase in the back pressure to bloodflow and increase in venous resistance. The increase in intrahepatic resistance was thought to occur via compression of the surface of the liver by descent of the diaphragm. Interestingly, this compressive effect appears to be independent of a generalized increase in intra-abdominal pressure. Thus, PEEP may lead to partitioning of blood from the right heart and pooling of blood in the splanchnic circulation.

A final pathway by which PEEP influences venous return is through its effect on renal hemodynamics and handling of water. PEEP leads to a decrease in renal bloodflow, glomerular filtration rate and urine flow, and an increase in fractional sodium reabsorption. These effects may also be secondary to PEEP's effects on renal perfusion pressure, as was recently demonstrated in a study in patients following renal transplant (a model of acute renal denervation) (7).

Right ventricular afterload: In the pulmonary arterial circulation some vessels are exposed to alveolar pressure (alveolar vessels) and others are not (extra-alveolar vessels). With the application of PPV and PEEP, transmural pressure (hence resistance) in the alveolar vessels increases. Conversely, with lung expansion the extra-alveolar vessels are tethered open and their resistance decreases. If the application of PEEP increases lung volume above functional residual capacity, the effects of alveolar vessel resistance predominate, and both pulmonary vascular resistance and right ventricular afterload will increase. This in turn may result in a decrease in right ventricular forward flow and stroke volume.

Summary: PPV and PEEP cause a decrease in right ventricular cardiac output through a decrease in preload and an increase in afterload. The net effects on left ventricular performance will depend on the loading and contractile state of that chamber.

\section{EFFECTS ON THE LEFT VENTRICLE}

Right ventricular output: With a decrease in right ventricular output there is a corresponding fall in left ventricular preload. In health, left ventricular output is predominately 


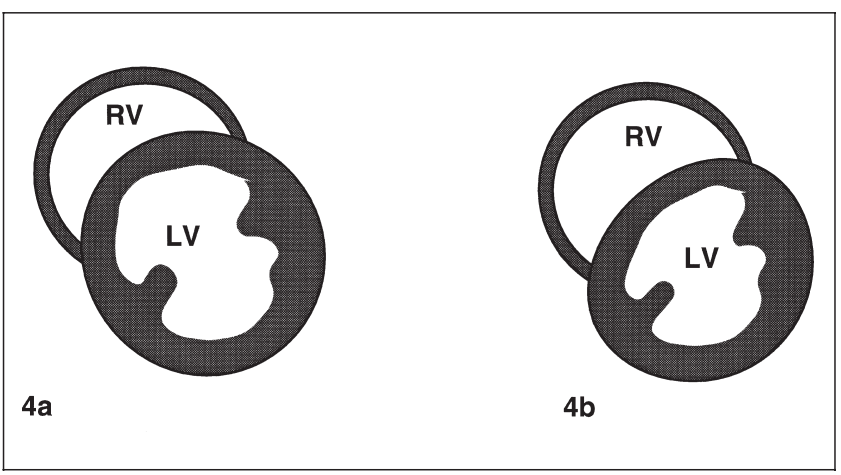

Figure 4) Septal shift. a Normally, the septal wall has a convexity towards the right ventricle $(R V)$ owing to a higher end-diastolic pressure in the left heart chamber. b During right ventricular overload the pressure in the right heart chamber increases and the curvature of the septum decreases. LV Left ventricle

preload-dependent. In normal volunteers cardiac output falls with the application of PEEP (8). In a failing, volume overloaded, dilated left ventricle, contractility may be decreased due to an increase in wall tension (which translates into increased myocardial work), and to mitral regurgitation due to a dilated mitral annulus leading to valvular incompetence. The addition of PEEP may be analogous to diuresis: by decreasing preload, PEEP decreases left ventricular volume, reduces wall tension and may reduce the severity of mitral regurgitation.

Septal shift: The ventricular septum separating the left and right chambers of the heart does not move appreciably during the diastolic filling phase. However, pressure and volume overload in one chamber may cause deviation of the septum towards the more compliant ventricle. When right ventricular afterload increases during the application of PEEP, there may be a corresponding increase in right ventricular volume. If the right ventricle dilates significantly the septum will shift towards the left chamber (Figure 4) (9). This in turn leads to a decrease in left ventricular volume and effectively reduces left ventricular preload. In general, this mechanism is more prominent during spontaneous inspiration when venous return is increased in the face of a simultaneous increase in right ventricular afterload. The net result is an increase in right ventricular volume and pressure, leading to a dramatic shift of the septum. This is one explanation for the development of pulsus paradoxus during spontaneous inspiration.

Transmural pressure: Because the left ventricle resides in the thorax, the pressure that the left heart must pump against is not simply mean arterial pressure. Rather, it is the difference between the mean systemic pressure and pleural pressure (mean pressure - pleural pressure). During spontaneous inspiration, pleural pressure falls and transmural pressure rises. In some settings this may be dramatic (upper airway obstruction, sleep apnea, Mueller manoeuvre and bronchospasm) and lead to a large increase in ventricular afterload. With the application of PEEP and PPV, pleural pressure becomes more positive and transmural pressure decreases (Figure 5). Thus, left ventricular afterload is effectively de-
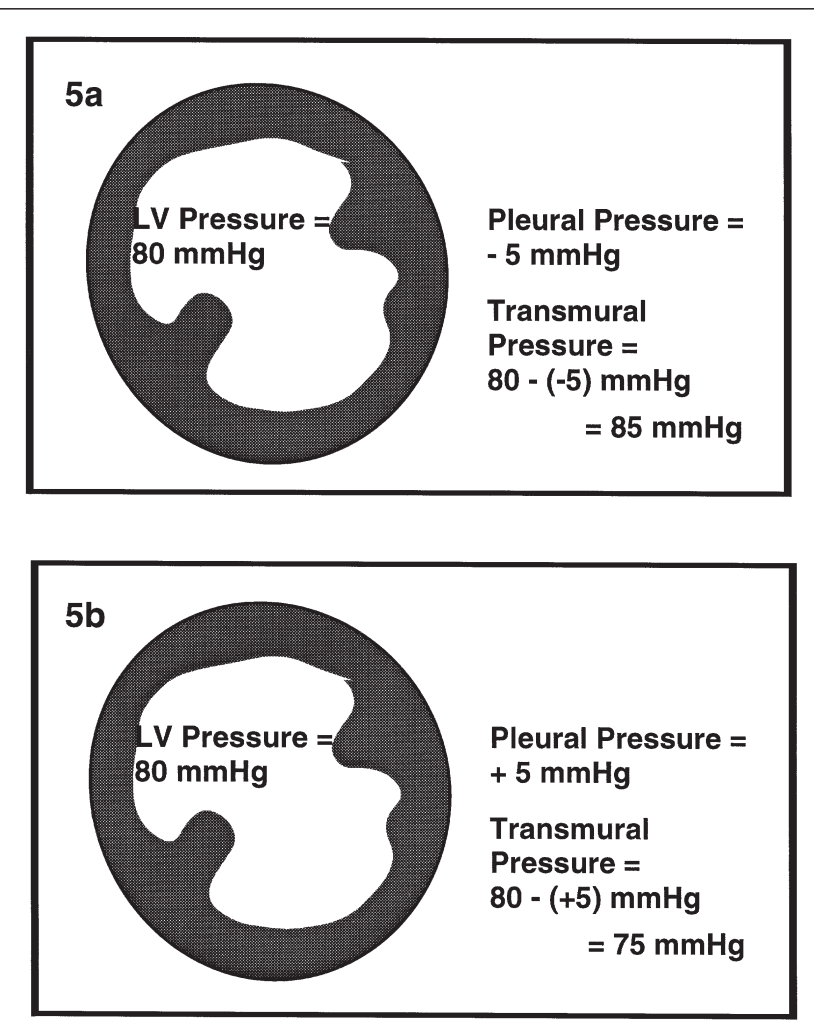

Figure 5) Transmural left ventricular (LV) pressure gradient. a During spontaneous breathing the pleural pressure is approximately $-5 \mathrm{mmHg}$. If the mean left ventricular pressure is $80 \mathrm{mmHg}$, then the transmural pressure that the left ventricle must eject against is $80-(-5)=85 \mathrm{mmHg}$. During the application of continuous positive airway pressure (CPAP), pleural pressure increases. b In this example pleural pressure is increased to $+5 \mathrm{mmHg}$ during CPAP and transmural pressure therefore is $80-(+5)=$ $75 \mathrm{mmHg}$

creased. The failing heart is exquisitely sensitive to the effects of afterload. An increase in afterload has a deleterious effect on stroke volume. Thus, PEEP may dramatically improve the left ventricular performance of a poorly contractile heart. A recent study by Huberfeld et al (10), however, throws this hypothesis into question. Monitoring juxtacardiac pressures during the application of continuous positive airway pressure in pigs, these investigators could not find any change in transmural pressures despite an improvement in cardiac output.

Work of breathing: Normally the respiratory muscles use less than $5 \%$ of the total $\mathrm{DO}_{2}$ (11). However, in the setting of congestive heart failure and an increase in lung water, the demand of the respiratory muscles for oxygen may exceed $25 \%$ of the total $\mathrm{DO}_{2}(12,13)$. This increased requirement results in higher cardiac demand and a reduction in $\mathrm{DO}_{2}$ to other organs. PPV may decrease work of breathing, reduce respiratory muscle oxygen requirements, improve $\mathrm{DO}_{2}$ and reduce cardiac work.

Afterload versus preload debate: There is persistent controversy in the literature as to whether the predominant effect of PEEP on the failing heart is due to changes in preload or 


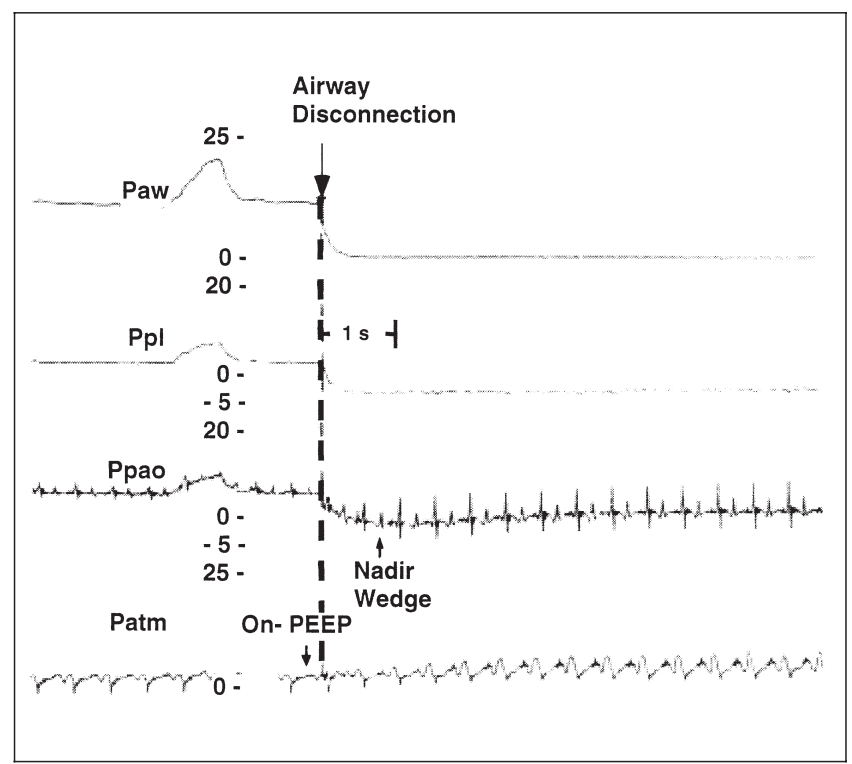

Figure 6) Recording of airway pressure (Paw), pleural pressure (Ppl), pulmonary arterial occlusion pressure (Ppao) and atmospheric pressure (Patm) both during $15 \mathrm{~cm} \mathrm{H}_{2} \mathrm{O}$ positive end-expiratory pressure (PEEP) and immediately after airway disconnection (first arrow). The time off PEEP is $1 \mathrm{~s}$. Nadir Ppao is measured at the second arrow. Note the gradual increase in Ppao with time. Reproduced with permission from reference 19

to changes in afterload $(8,10,14)$. Practically, it is difficult to tease out the independent effects of preload and afterload, owing to limitations of animal models, methods of measurement and ventricular interdependence. Often the response to a given level of PEEP in an individual patient is unpredictable. This is analogous to the variation seen among patients in their response to diuretics and afterload-reducing agents. A trial and error approach may be required to determine the optimal level of PEEP.

Irrespective of which of the above mechanisms predominate, the potential benefits of PEEP on cardiac performance in patients with left ventricular dysfunction are gradually being recognized. Continuous positive airway pressure by mask has been shown to reduce the requirement for intubation and ventilation in patients with acute congestive heart failure (15) and to improve stroke volume in patients with advanced, stable cardiac dysfunction (16).

Many are also familiar with the situation in the intensive care unit with patients who are extubated following treatment for congestive failure and rapidly develop recurrent pulmonary edema. Although this may be due, in part, to increased cardiac stress from the additional work of breathing, it is more likely the result of the loss of PEEP's beneficial effects on filling pressures and afterload in the failing heart (17).

\section{MONITORING THE EFFECTS OF PEEP AND PPV}

As mentioned previously, following the changes in $\mathrm{DO}_{2}$ in an individual patient remains an important method of determining the net hemodynamic effect of PPV and PEEP. At the bedside one is also often faced with the situation of attempting to determine filling pressures in a patient with high levels of PEEP or mean airway pressure. Several limitations to the measurement of cardiac pressures need to be recognized. First, no single number is diagnostic; one needs to follow trends in filling pressures and observe changes with alterations in therapeutic strategies. Second, pressure is used as an estimate of volume; unless compliance remains constant, a change in pressure may not reflect a parallel change in volume. As an example, as left ventricular compliance is decreased, end-diastolic wedge pressure increases but ventricular volume (preload) decreases. Third, filling pressures need to be referenced to the pressure surrounding the chamber of interest. Pleural pressure may vary throughout the thorax and different regions of the heart and great vessels. Thus, even if one attempts to measure pleural pressure (eg, with the aid of an esophageal balloon) it may not accurately reflect the pressure that the ventricle or chamber is actually experiencing.

In an attempt to minimize the effects of positive pleural pressure on hemodynamic pressure recordings, some have suggested reducing the measured wedge pressure by some fraction of the PEEP. This approach is likely too simplistic given the foregoing discussions of intrathoracic pressure and difficulties in determining regional or juxtacardiac pressures. Others have advocated measuring the wedge pressure following discontinuation of PEEP. A major concern surrounding this approach is that measurement when PEEP is interrupted is removed from the hemodynamic milieu that exists when PEEP was present. With the discontinuation of PEEP there is an increase in venous return to the right ventricle, and an increase in blood returning to the left heart owing to a decrease in alveolar pressure and lung volume. However, Pinsky et al (18) and Carter et al (19) argue that the pressure nadir of the 'wedge' tracing seen $2 \mathrm{~s}$ following the discontinuation of PEEP accurately reflects left atrial filling pressure because it occurs before the development of significant fluid shifts (Figure 6). As emphasized in their discussion and in subsequent correspondence, the change in right atrial pressure following the removal of PEEP may be a better reflection of the extracardiac pressure contributed by PEEP. Practically, for the physician caring for a patient at the bedside, these arguments serve to emphasize the need to follow trends in vascular pressures as opposed to individual values per se.

\section{CONCLUSIONS}

PPV and PEEP lead to alterations in stroke volume through changes in lung volume and, second, through alterations in intrapleural and intra-abdominal pressure. The net effect on ventricular performance is influenced by the loading conditions and contractile function of the heart. In addition, the presence of lung disease may alter these relationships. These factors make the net cardiovascular response to changes in PPV and PEEP in an individual patient difficult to predict. Monitoring changes in hemodynamics and oxygen delivery are essential to determine adequately the cardiovascular consequences of PPV in the critically ill patient. 


\section{REFERENCES}

1. Buda AJ, Pinsky MR, Ingels NB, et al. Effect of negative intrathoracic pressure on left ventricular performance. N Engl J Med 1979;301:453-9.

2. Leithner C, Podolsky A, Globits S, et al. Magnetic resonance imaging of the heart during positive end-expiratory pressure ventilation in normal subjects. Crit Care Med 1994;22:426-32.

3. Sylvester JT, Goldberg HS, Permutt S. The role of the vasculature in the regulation of cardiac output. Clin Chest Med 1983;4:111-26.

4. Fessler HE, Brower RG, Shapiro EP, Permutt S. Effects of positive end-expiratory pressure and body position on pressure in the thoracic great veins. Am Rev Respir Dis 1993;148:1657-64.

5. Takata M, Wise R, Robotham JL. Effects of abdominal pressure on venous return: abdominal vascular zone conditions. J Appl Physiol 1990;69:1962-72.

6. Brienza N, Revelly J-P, Ayuse T, Robotham JL. Effects of PEEP on liver arterial and venous blood flows. Am J Respir Crit Care Med 1995; 152:504-10.

7. Jacob LP, Chazalet J-JA, Payen DM, et al. Renal hemodynamic and functional effect of PEEP ventilation in human renal transplantations. Am J Respir Crit Care Med 1995;152:103-7.

8. Bradley TD, Holloway RM, McLaughlin PR, Ross BL, Walters J, Liu PP. Cardiac ouput response to continuous positive airway pressure in congestive heart failure. Am Rev Respir Dis 1992;145:377-82.

9. Brinker JA, Weiss JL, Lappé DL, et al. Leftward septal displacement during right ventricular loading in man. Circulation 1980;61:626-33.

11. Nunn JF. Applied Respiratory Physiology. London: Butterworth, 1987:104.

12. Stock MC, Downs JB, Betts RK, Frolicher DA, Howie MB, Tallman
RD. Oxygen consumption during spontaneous ventilation with acute lung injury in anesthetized pigs. Am Rev Respir Dis 1988;137:790-5.

13. Field S, Kelly SM, Macklem PT. The oxygen cost of breathing in patients with cardiorespiratory disease. Am Rev Respir Dis 1982;126:9-13.

10. Huberfeld SI, Genovese J, Tarasiuk A, Scharf SM. Effect of CPAP on pericardial pressure and respiratory system mechanics in pigs. Am J Respir Crit Care Med 1995;152:142-7.

14. Marini JJ, Culver BH, Butler J. Mechanical effect of lung distention with positive pressure on cardiac function. Am Rev Respir Dis 1981;124:382-6.

15. Rasanen J, Heikkila J, Downs J, Nikki P, Vaisanen I, Viitanen A. Continuous positive airway pressure by face mask in acute cardiogenic pulmonary edema. Am J Cardiol 1985;55:296-300.

16. Naughton MT, Liu PP, Benard DC, Goldstein RS, Bradley TD. Treatment of congestive heart failure and Cheyne-Stokes respiration during sleep by continuous positive airway pressure. Am J Respir Crit Care Med 1995;151:92-7.

17. Lemaire F, Teboul J-L, Cinotti L, et al. Acute left ventricular dysfunction during unsuccessful weaning from mechanical ventilation. Anesthesiology 1988;69:157-60.

18. Pinsky M, Vincent J-L, DeSemet JM. Estimating left ventricular filling pressure during positive end-expiratory pressure in humans. Am Rev Respir Dis 1991;143:25-31.

19. Carter R, Snyder J, Pinsky M. LV filling pressure during PEEP measured by nadir wedge pressure after airway disconnection. Am J Physiol 1985;249:H770-6. 


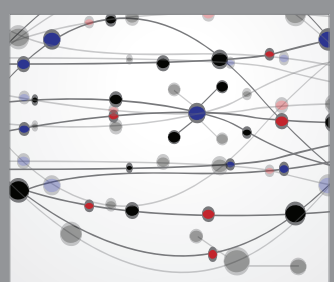

The Scientific World Journal
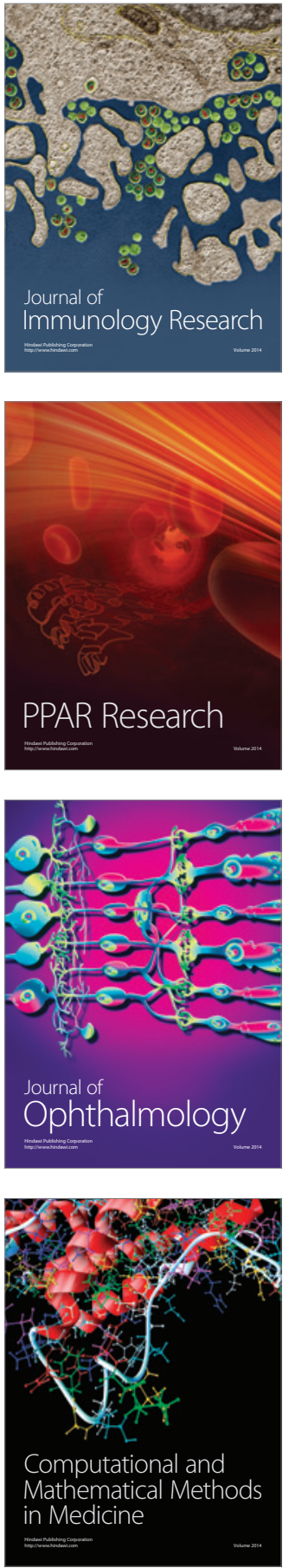

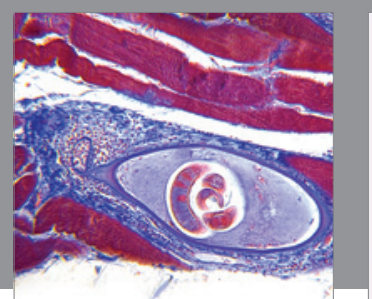

Gastroenterology Research and Practice

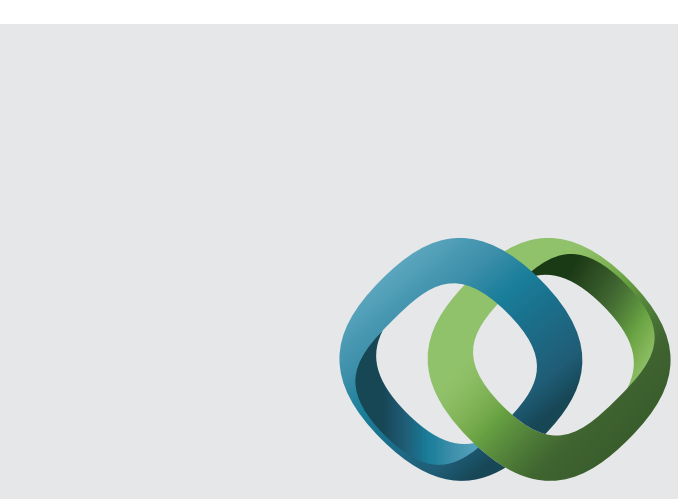

\section{Hindawi}

Submit your manuscripts at

http://www.hindawi.com
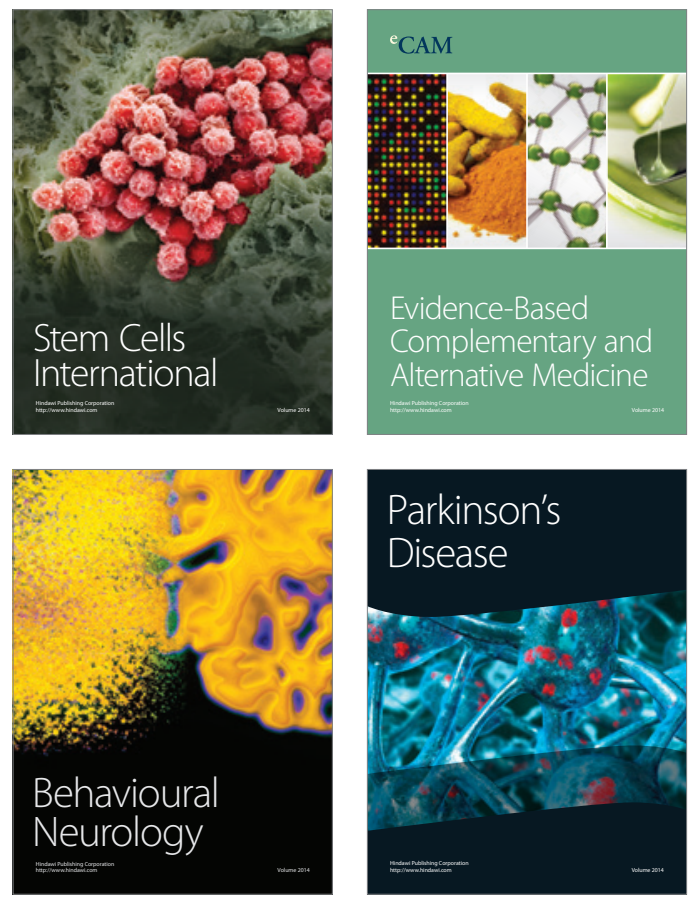
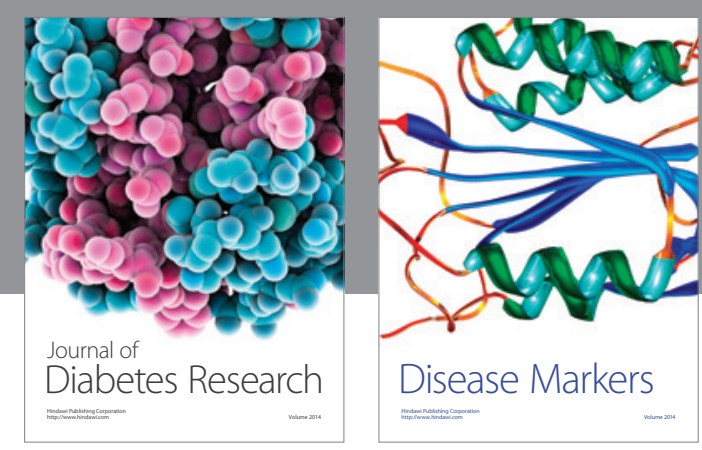

Disease Markers
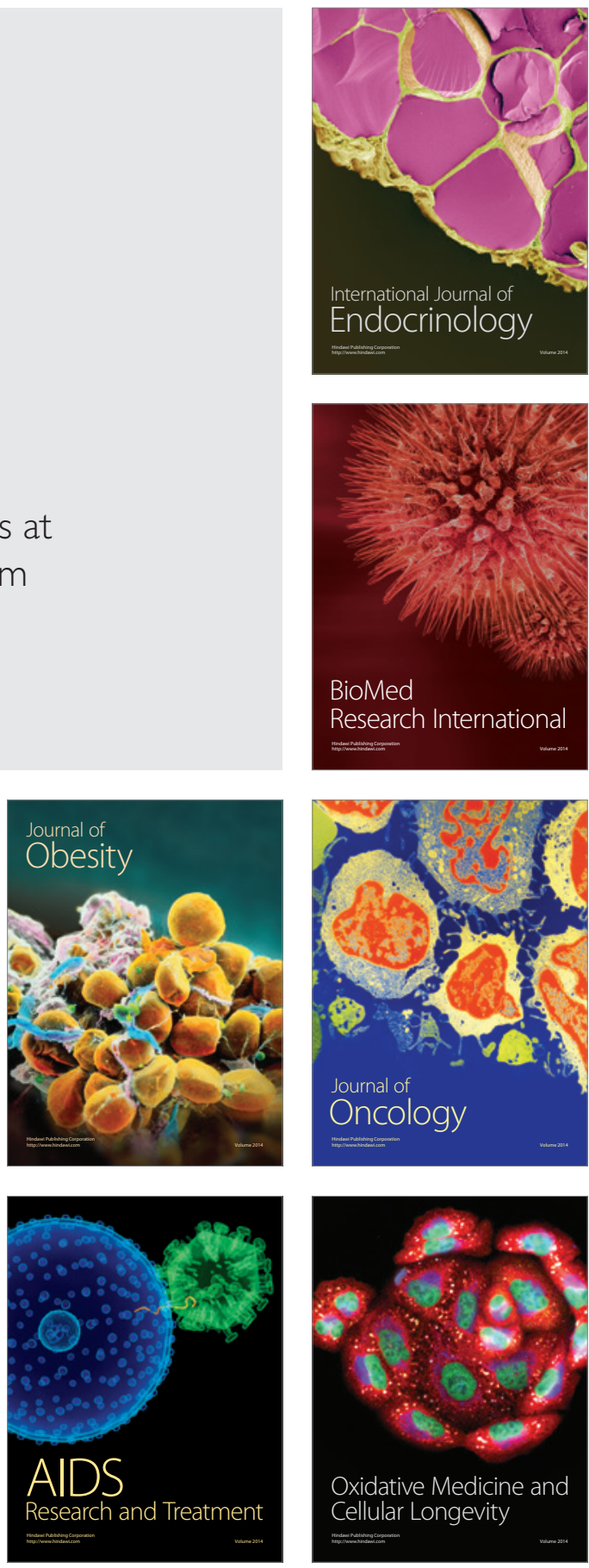